\title{
Endocarditis de Libman-Sacks de válvulas nativas derechas en el contexto de síndrome antifosfolípido y lupus eritematoso sistémico. ¡Cuando el tratamiento marca la diferencia!
}

Alma S. Arrioja Salazar*

Luis Emiro Velazco C**

\section{Correspondencia}

Alma Arrioja

almasthelaarrioja@gmail.com

*Cardiólogo-ecocardiografista. Coordinadora Ad Honorem de Docencia e Investigación del Sanatorio Trinidad Ramos Mejía. Buenos Aires, Argentina

${ }^{* *}$ Cardiólogo-Ecocardiografista. Jefe del Servicio de Ecocardiografía. ASCARDIO. Barquisimeto-Venezuela

\author{
Recibido: 04/01/2020 \\ Aceptado: 04/01/2020 \\ En línea: 02/03/2020
}

Citar como: Arrioja-Salazar AS, Velazco LE. Endocarditis de Libman-Sacks de válvulas nativas derechas en el contexto de síndrome antifosfolípido y lupus eritematoso sistémico. ¡Cuando el tratamiento marca la diferencia! Rev Ecocar Pract (RETIC). 2020 (Mar); 3 (1): 19-22. doi: 10.37615/retic.v3n1a5.

Cite this as: Arrioja-Salazar AS, Velazco LE. Libman-Sacks endocarditis of right native valves in the context of antiphospholipid syndrome and systemic lupus erythematosus. When the treatment makes a difference! Rev Ecocar Pract (RETIC). 2020 (Mar); 3 (1): 19-22. doi: 10.37615/retic.v3n1a5

\section{Palabras clave}

$\triangleright$ Endocarditis

$\triangleright$ Libman-Sacks

$\triangleright$ Antifosfolípido

$\triangleright$ Lupus

\begin{abstract}
RESUMEN
Se presenta el caso de una mujer de 54 años con síndrome antifosfolípido en terapia irregular, que ingresa por disnea de esfuerzo que progresó a ortopnea. La ecocardiografía transtorácica evidenció cardiopatía dilatada con disfunción sistólica biventricular, hipertensión pulmonar y masas relacionadas con válvulas pulmonar y tricúspide sin movimiento autonómico. Los cultivos y el recuento de la serie blanca estaban normales, con alteración de las pruebas del SAF, haciéndose además diagnóstico de lupus eritematoso sistémico. Se inició terapia para insuficiencia cardíaca, esteroides, rituximab y anticoagulantes, mejorando la clínica. La ecocardiografía control mostró remisión de las masas tricúspides y similares dimensiones de la masa pulmonar.
\end{abstract}

ABSTRACT
We report the case of a 54-year-old woman with antiphospholipid syndrome in irregular therapy, admitted due
to exertional dyspnea and orthopnea. The transthoracic echocardiogram showed dilated cardiomyopathy with
biventricular systolic dysfunction, pulmonary hypertension and masses related to the pulmonary and tricuspid
valves without autonomic movement. The crops and white count were normal, with alteration of the SAF test. In
addition, SLE was diagnosed. It was started therapy for heart failure, steroids, rituximab and anticoagulation, with
improving of the symptoms. The control echocardiogram showed remission of the tricuspid masses and similar
dimensions of the pulmonary mass.

Presentación del caso

Llamativamente, se observaron masas ecoheterogéneas relacionadas con las válvulas nativas derechas:

- En la superficie ventricular de la valva pulmonar relacionada con la válvula aórtica se muestra una masa ovalada de bordes irregulares, base ancha, con movilidad acorde al ciclo cardíaco, con unas medidas de $8 \times 7 \mathrm{~mm}$.

- Otras tres masas de mayor tamaño, ovales, de bordes regulares, poco móviles, en la superficie ventricular de las valvas tricúspides, con medidas de $16 \times 7 \mathrm{~mm}$ (valva septal), $11 \times 8 \mathrm{~mm}$ (valva anterior) y $10 \times 5 \mathrm{~mm}$ (valva posterior). De estas tres últimas masas, la relacionada con la valva anterior era la más hipoecoica.

Por estos hallazgos se solicitaron anticuerpos antifosfolípidos y anticardiolipinas (aCL) (que estaban positivos), proteína C reactiva y velocidad de eritrosedimentación (que estaban elevadas), hemocultivos negativos, hemoglobina de $11,5 \mathrm{~g} / \%$ y glóbulos blancos en $9.500 / \mathrm{mm}^{3}$. Adicionalmente, se hizo el diagnóstico de lupus eritematoso sistémico (LES) 
Con la presunción diagnóstica de endocarditis bacteriana se inició terapia para insuficiencia cardíaca y glucocorticoides a altas dosis, rituximab y anticoagulación oral.

La paciente presentó franca mejoría clínica y regresó tras 5 meses para un control ecocardiográfico donde se observó mejoría de la función sistólica biventricular, disfunción diastólica grado I del VI y PSP en $45 \mathrm{mmHg}$. Se evidenció similar tamaño de la masa pulmonar $(8 \times 6 \mathrm{~mm})$ y desaparición de las masas de las valvas tricúspides.

Por todos los hallazgos patológicos ecocardiográficos previamente descritos y con remisión significativa del tamaño de las masas intracardíacas tras la terapia para la patología de base, se puede concluir el caso con diagnóstico de endocarditis bacteriana de Libman-Sacks en válvulas nativas derechas en contexto de SAFy LES.

\section{Estudio por imagen}

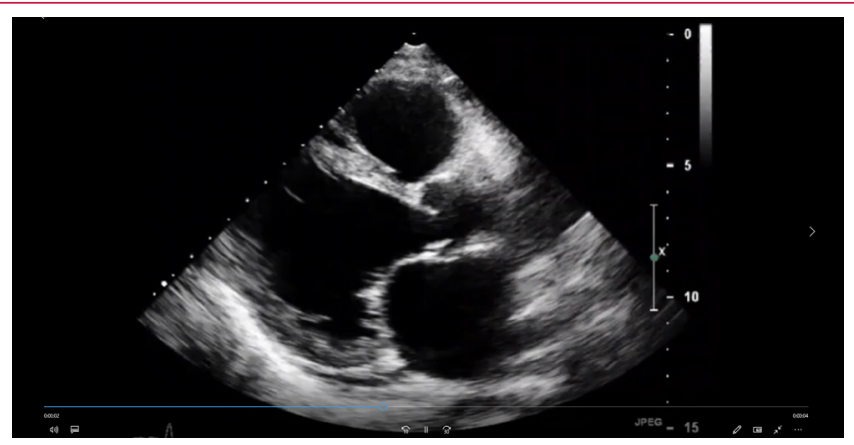

Vídeo 1. Ecocardiografía transtorácica (ETT) paraesternal de eje largo con cardiopatía dilatada, disfunción sistólica biventricular y dilatación leve de aurícula izquierda

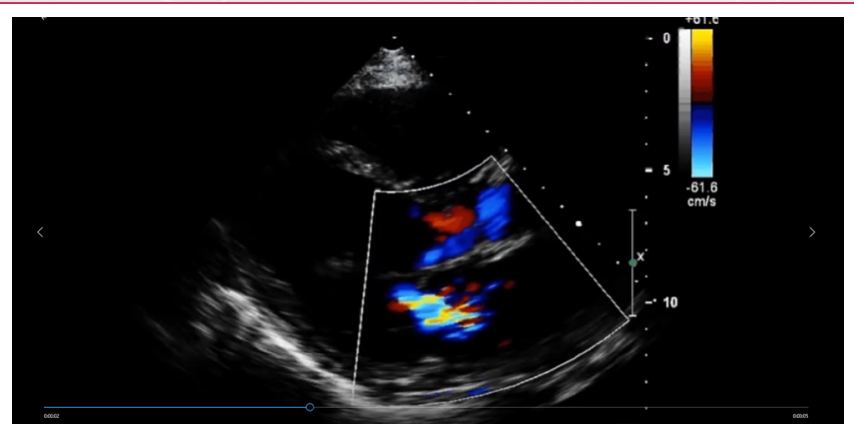

Vídeo 2. ETT paraesternal de eje largo con color que evidencia insuficiencia mitral moderada (funcional)

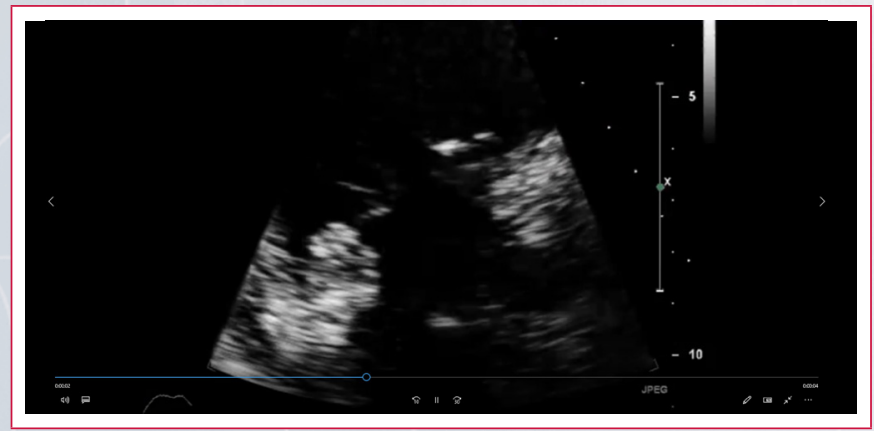

Vídeo 3. ETT, vista del tracto de entrada del VD (TEVD) en modo zoom con evidencia de masa en cara ventricular de valva posterior tricúspide

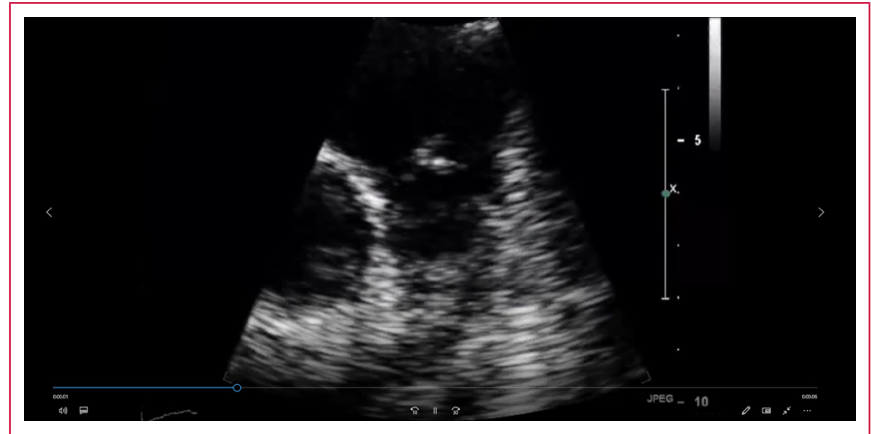

Vídeo 4. ETT vista de eje corto de grandes vasos en modo zoom enfocado en válvula pulmonar con evidencia de masa en cara ventricular de valva relacionada con válvula aórtica

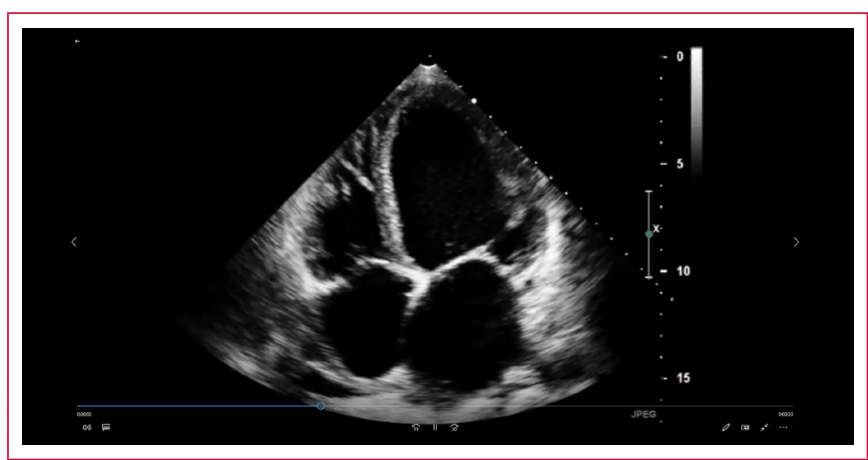

Vídeo 5. ETT plano apical de cuatro cámaras, cardiopatía dilatada con disfunción sistólica biventricular, contraste espontáneo en el VI, masa en cara ventricular de valva septal tricúspide, derrame pericárdico leve y derrame pleural izquierdo

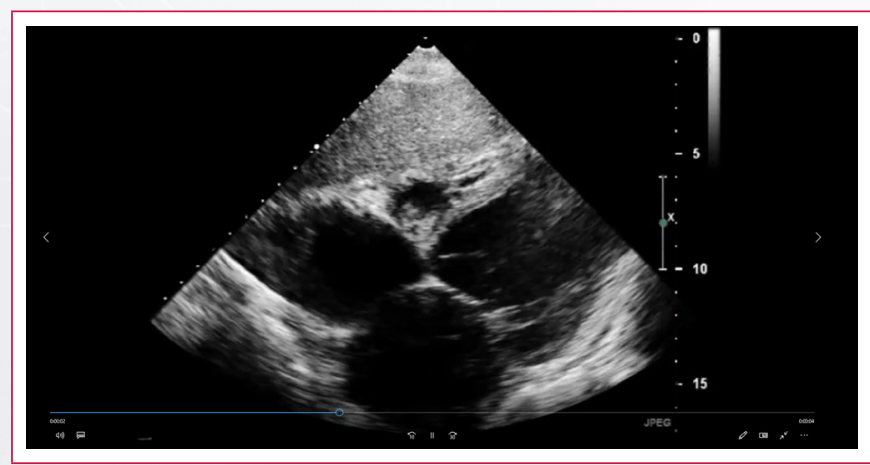

Vídeo 6. ETT plano subcostal de cuatro cámaras, cardiopatía dilatada con disfunción sistólica biventricular, masas en cara ventricular de valva septal y anterior tricúspide y derrame pericárdico leve

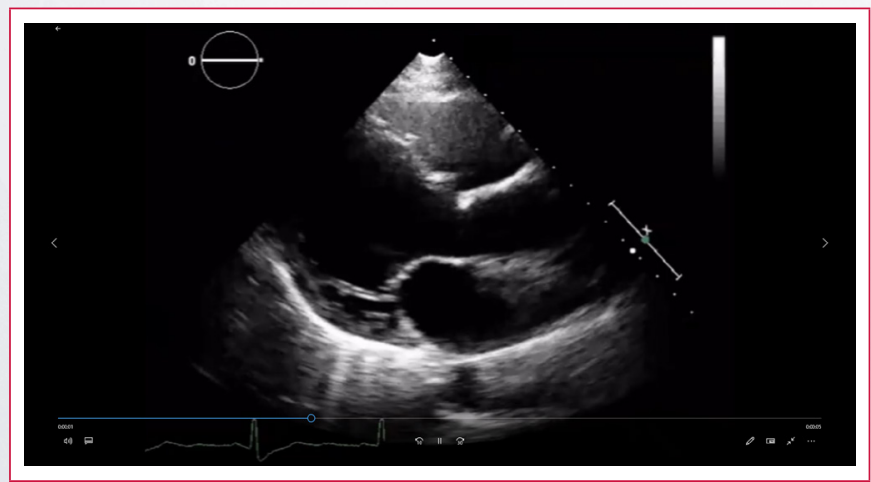

Vídeo 7. ETT paraesternal de eje largo tras 5 meses en tratamiento, con evidencia de cardiopatía dilatada y mejoría de la función sistólica biventricular 


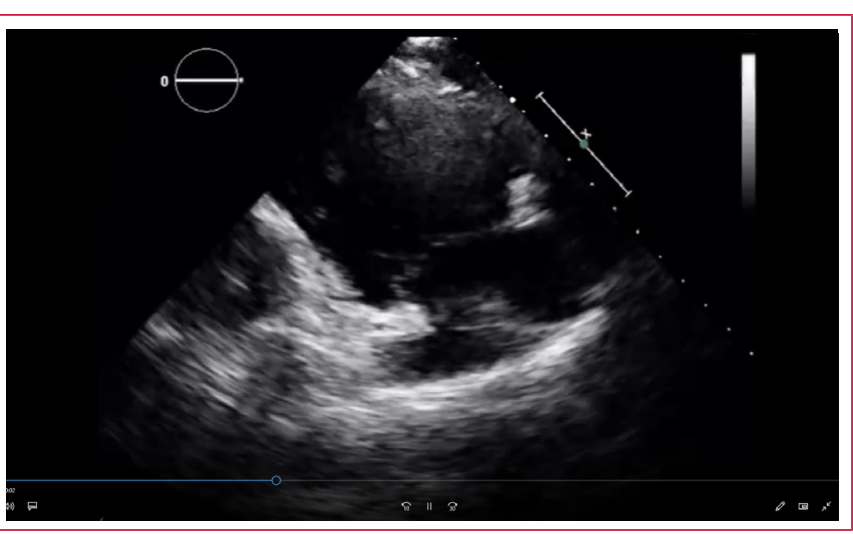

Vídeo 8. ETT vista del TEVD sin evidencia de la masa que previamente se encontraba en cara ventricular de valva posterior tricúspide

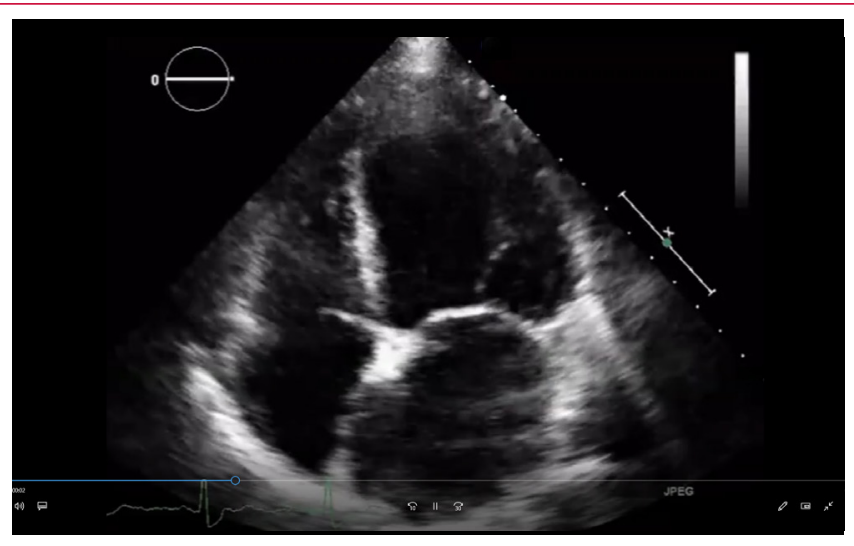

Vídeo 9. ETT, plano apical de cuatro cámaras con cardiopatía dilatada y mejoría de la función sistólica biventricular, remisión de la masa en valva septal tricúspide, ausencia de derrame pericárdico y pleural

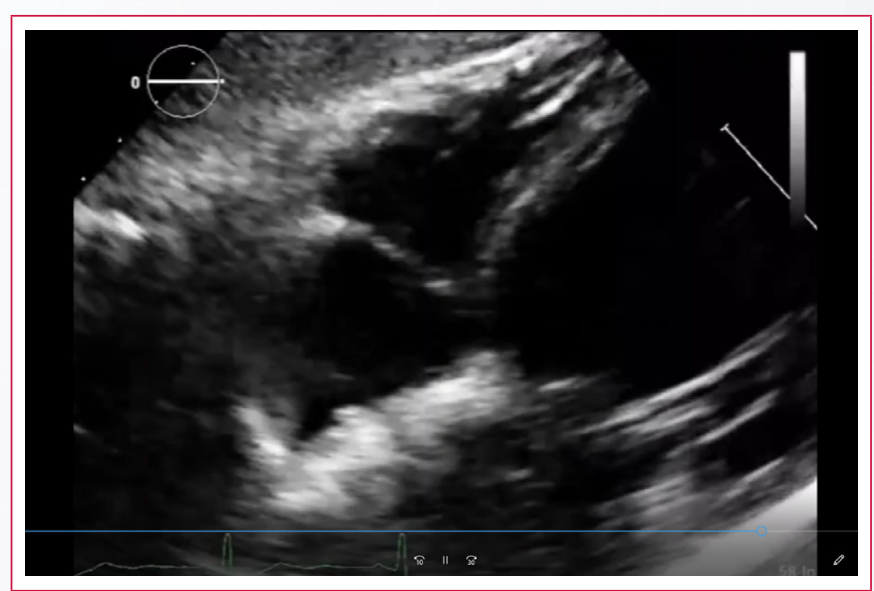

Vídeo 10. ETT, subcostal de cuatro cámaras donde se evidencia remisión de las masas evidenciadas previamente en valva septal y anterior tricúspide
EI SAF secundario aumenta la frecuencia y la gravedad de la enfermedad valvular cardíaca en el LES. En el caso de esta paciente el diagnóstico de SAF se hizo primero ${ }^{(3,4,5)}$.

Ecocardiográficamente, las lesiones de la ELS suelen ser sésiles, regulares, de base ancha, con poca movilidad, sin movimiento errático, ubicadas en el lado ventricular de las válvulas auriculoventriculares, en el lado vascular de las válvulas sigmoideas o en el endocardio vascular, tales características las diferencian de las típicas vegetaciones infecciosas.

La patogenia de las lesiones valvulares en la ELS implica la formación de fibrina, plaquetas y trombos en la válvula alterada, el daño endotelial, depósito e inmunoglobulinas, aCL y complemento, lo que conduce a fibrosis, engrosamiento, cicatrización, distorsión y disfunción valvular. Aunque la afección suele ser leve, en algunos pacientes las anomalías pueden ser mayores, causar embolización y regurgitación valvular severa que requieren cirugía ${ }^{(1,6,7,8)}$.

La "hipótesis de los 2 impactos" descrita por Bordin et al., plantea que el estado protrombótico generado por los anticuerpos aCL (primer evento) favorecería (tras un segundo evento como cirugía cardíaca, embarazo, etc.), la formación del trombo sobre un endotelio valvular previamente inflamado y actuar como desencadenante del cuadro de endocarditis sobre una válvula que siendo susceptible previamente, aumenta la potencial afección $n^{(1,2,3,4)}$.

Aproximadamente el $10 \%$ de los pacientes con LES desarrollan insuficiencia valvular severa asociada con altos niveles de anticuerpo aCL, síntomas de insuficiencia cardíaca y disfunción ventricular que amerita cirugía ${ }^{(3,4,5)}$. Vianna et al. informan que las lesiones valvulares son más severas en pacientes con SAF secundario ${ }^{(5)}$.

La cirugía se reserva para los casos con disfunción valvular severa y grandes vegetaciones embolígenas. Aunque el tratamiento con esteroides puede reducir las lesiones, no es suficiente para erradicar el riesgo embólico y la anticoagulación no debería cuestionarse $e^{(5,6)}$

El uso de esteroides ha provocado una importante reducción de la incidencia de ELS, sin embargo, su frecuencia actual alcanza 10-35\% de los casos. En este caso hubo importante remisión de las masas tricúspides con el tratamiento que recibió, persistiendo la lesión pulmonar. Pese a ello, sus manifestaciones clínicas suelen ser leves y suele transcurrir inadvertida.

\section{Conclusión}

La ELS debe incluirse en el diagnóstico diferencial del paciente con SAF y LES con presencia de masas intracardíacas que carecen del típico movimiento errático de las vegetaciones infecciosas, en especial si existen anticuerpos aCL positivos con cultivos negativos. Aunque la disfunción valvular es rara, cuando se produce, el tratamiento con esteroides y terapia específica del SAF/LES puede reducir las lesiones y diferir la necesidad de reemplazo valvular. Dado el riesgo embólico, la anticoagulación y vigilancia clínica y de imagen son fundamentales.

\section{Discusión}

La endocarditis de Libman-Sacks (ELS) es una de las presentaciones cardíacas más frecuentes del lupus eritematoso sistémico (LES) y del síndrome antifosfolípidos (SAF) que típicamente afecta a la válvula aórtica o mitral, la participación de la válvula tricúspide y pulmonar es altamente inusual (menos del 10\% de los casos documentados) $)^{(1,2,6,7,8)}$.

\section{Ideas para recordar}

- La ELS es frecuente en pacientes con SAF y LES y debe pensarse ante masas intracardíacas con cultivos negativos en pacientes sin fiebre.

- Las válvulas más afectadas son las izquierdas.

- El tratamiento de la patología de base suele lograr reducción del tamaño de las masas y podría mejorar la disfunción ventricular. 


\section{Bibliografía}

1. Moyssakis I, Tektonidou MG, Vassilliou VA, et al. Libman-Sacks endocarditis in systemic lupus erythematosus: Prevalence, associations, and evolution. Am J Med 2007; 120: 636-642.

2. Sharma J, Lasic Z, Bornstein A, et al. Libman-Sacks endocarditis as the first manifestation of systemic lupus erythematosus in an adolescent, with a review of the literature. Cardiol Young 2013; 23: 1-6.

3. Bordin G, Boldorini R, Meroni PL. The two hit hypothesis in the antiphospholipid syndrome: Acute ischaemic heart involvement after valvular replacement despite anticoagulation in a patient with secondary APS. Lupus 2003; 12: 851-853.

4. Vinales KL, Gopalan RS, Lanza LA, et al. Unusual case of nonbacterial thrombotic endocarditis attributable to primary antiphospholipid syndrome. Circulation 2010; 122: e459-460.
5. Vianna JL, Khamashta MA, Ordi-Ros J, et al. Comparison of the primary and secondary antiphospholipid syndrome: a European multicenter study of 114 patients. Am J Med 1994; 96 (1): 3-9.

6. Laufer J, Frand M, Milo S. Valve replacement for severe tricuspid regurgitation caused by Libman-Sacks endocarditis. Br Heart J 1982; 48 (3): 294-297.

7. Bhimani AA, Hoit BD. Extensive nonbacterial thrombotic endocarditis isolated to the tricuspid valve in primary antiphospholipid syndrome. J Am Echocardiogr 2010; 23 (1): 107.e5-6.

8. Ziporen L, Goldberg I, Arad M, et al. Libman-Sacks endocarditis in the antiphospholipid syndrome: immunopathologic findings in deformed heart valves. Lupus 1996; 5 (3):196-205. 